\title{
Femtosecond Laser Spectroscopy and Singlet Fission
}

\section{Gagik G Gurzadyan*}

Institute of Artificial Photosynthesis, State Key Laboratory of Fine Chemicals, Dalian University of Technology, Dalian, China

*Corresponding author: Gagik G Gurzadyan, Institute of Artificial Photosynthesis, State Key Laboratory of Fine Chemicals, Dalian University of Technology, Dalian, China, Tel: +86-188-4095 4612; E-mail: gurzadyan@ntu.edu.sg

Received date: May 31, 2016, Accepted date: June 3, 2016, Published date: June 3, 2016

Copyright: $\odot 2016$ Gurzadyan GG. This is an open-access article distributed under the terms of the Creative Commons Attribution License, which permits unrestricted use, distribution, and reproduction in any medium, provided the original author and source are credited.

\section{Introduction}

Singlet exciton fission or singlet fission (SF) is a process in which a singlet excited molecule, usually in a densely packed organic solid or in a polymer, shares its energy with a neighboring molecule in its electronic ground state, both molecules forming a pair of triplet states in a spin allowed process. SF was first proposed in 1965 to explain the delayed fluorescence in anthracene crystal [1]. It was further proven by the magnetic field effect on tetracene crystal $[2,3]$. Interest towards SF rises in recent years due to its potential applications in photovoltaics. SF may dramatically increase efficiency of the solar cells.

$\mathrm{SF}$ is an analogue of the Multiple-Exciton Generation (MEG) in the organic materials. A comprehensive review on SF is given by Smith and Michl [4,5].

Femtosecond laser spectroscopy is a powerful method for studying the kinetics of SF. Transient absorption allows monitoring with femtosecond time resolution decay of the excited singlet state and corresponding rise of the triplet state, as well as existence of intermediate states. Thus the rate constant of the singlet fission can be determined and the mechanism of SF can be resolved.

\section{Singlet fission: rate constant}

Transient absorption spectra in rubrene single crystal were measured under $500 \mathrm{~nm}$ excitation. Two positive transient bands with maxima at 444 and $510 \mathrm{~nm}$ were attributed to singlet-singlet and triplet-triplet absorption, respectively [6]. Transient band at $444 \mathrm{~nm}$ decays and at $510 \mathrm{~nm}$ develops biexponentially: $\tau_{1}=2.3 \mathrm{ps}, \tau_{2}=23$ ps. An isosbestic point at $455 \mathrm{~nm}$ is an indication of a single transformation process, in which singlet states are converted into triplet states $\left(\mathrm{S}_{1} \rightarrow \mathrm{TT}\right)$. The triplet states form with two rate constants, $\mathrm{k}_{1}=4.3^{\star} 10^{11} \mathrm{~s}^{-1}$ and $\mathrm{k}_{2}=4.3^{*} 10^{10} \mathrm{~s}^{-1}$, which correspond to the direct fission from the upper vibrational states of $S_{1}$ and thermally activated fission from the relaxed $S_{1}$, respectively.

\section{Efficiency of singlet fission}

SF is a spin allowed highly efficient process; it can reach $200 \%[7,8]$. Efficiency can be determined from the quenching of the excited state lifetime in crystals compared with the solution; in rubrene single crystal was obtained $99.99 \%$ efficiency of SF, which corresponds to triplet formation with $199.98 \%$ [6,9]. Moreover, SF is a linear optical process, i.e. low intensity sunlight may be used for this purpose.

\section{Singlet fission: temperature dependence}

Apart from the transient absorption spectroscopy, time resolved fluorescence (up-conversion and time-correlated single photon counting) also provides a direct way for studying SF. For example, in rubrene single crystal SF is a thermally activated reaction, therefore it is temperature dependent. The quenching of fluorescence is mainly due to SF, and the fluorescence lifetime reflects the rate of SF.

It was demonstrated that at low temperatures $80 \mathrm{~K}$, SF in rubrene can be fully supressed [9]. This was however not observed in tetracene [10].

\section{Singlet fission from upper vibrational states of $S_{1}$}

Excitation wavelengths of 400, 500 and $550 \mathrm{~nm}$ were used in order to excite different vibrational states of the first excited singlet state $S_{1}$ in rubrene crystal. SF rate was found to be pump wavelength dependent [11]. The triplet states form faster with decreasing pump wavelength (i.e., increasing pump photon energy). It points out that singlet fission can proceed directly from the upper vibrational states of $S_{1}$. Singlet fission is faster from the upper excited states $(2.3 \mathrm{ps})$ since it is exoergic.

\section{Singlet fission from upper excited singlet states $\mathrm{S}_{\mathrm{N}}$}

Singlet fission was observed to proceed directly from upper excited singlet states SN, after excitation with $250 \mathrm{~nm}$ [11]. It can be considered as violation of Kasha rule (and corresponding analogue in spectroscopy: Vavilov law), which states that all photophysical and photochemical processes necessarily proceed from the lowest excited electronic states $\mathrm{S}_{1}$ or $\mathrm{T}_{1}$. The relation between the direct singlet fission rate lnk fiss versus the pump photon energy undergoes a linear increase, i.e. follows the Arrhenius law.

\section{Singlet fission: two-photon excitation}

Two-photon excited state relaxation processes in rubrene single crystal were studied under excitation with $=750 \mathrm{~nm}$, i.e. no linear absorption [11]. Although the two-photon excited states have different symmetries relative to the one-photon excited states, SF was still observed. The rate of SF was $\mathrm{k}=6^{*} 10^{11} \mathrm{~s}^{-1}$.

Thus with two photons of $1.6 \mathrm{eV}$ two triplet states with energies of $1.14 \mathrm{eV}$ can be populated. Hence via SF triplet states can be formed not only with high quantum, but also high energy conversion efficiency. Note that the quantum yield of two-photon process is constant and does not depend on the intensity of exciting light.

\section{Singlet fission: consecutive two-quantum (two-step) excitation}

SF can proceed also after excitation of the high-lying excited electronic states via consecutive singlet-singlet absorption: $S_{0} \rightarrow S_{1} \rightarrow$ $\mathrm{S}_{\mathrm{N}}$. In this case much lower radiation intensities are required compared 
with the above described two-photon excitation. This was demonstrated for the organic single crystal $\alpha$-perylene [12].

In a-perylene SF has at a threshold: $3.51 \mathrm{eV}(353 \mathrm{~nm})$ [13]. The competing reaction is a fast excimer formation. In [12] efficient SF was observed under high intensity femtosecond laser excitation both below and above this threshold. Below the threshold, it is explained in terms of both two-photon absorption (simultaneous absorption of two photons) and consecutive two-quantum absorption. Due to the dimeric structure of $\alpha$-perylene the observed fission occur ultrafast: $\ll 50$ fs.

\section{D coherent laser spectroscopy}

Series of pentacene derivatives were studied by using ultrafast twodimensional electronic spectroscopy in order to unravel the origin of the states involved in fission [14]. Obtained data indicate the crucial role of vibrational degrees of freedom coupled to electronic excitations that facilitate the mixing of multiexcitonic states with singlet excitons. The resulting manifold of vibronic states drives sub-100 fs fission with unity efficiency.

\section{Ultrafast microscopy}

Direct visualization of exciton transport in single tetracene crystals using transient absorption microscopy with $200 \mathrm{fs}$ time resolution and $50 \mathrm{~nm}$ spatial precision was reported [15]. These measurements reveal a new singlet-mediated transport mechanism for triplets, which leads to a factor of 10 enhancement in effective triplet exciton diffusion.

\section{Singlet fission in solution}

SF was reported to yield triplet formation with $200 \%$ efficiency in solutions of bis(triisopropylsilylethynyl (TIPS)) pentacene. This is a relatively slow process (ns scale) since triplet formation rates are controlled by the diffusion limit [16]. One main direction of singlet fission material design is to synthesise a properly coupled dimer structure, aiming to achieve high efficient intramolecular SF in dilute solution or low crystallinity films from simple solution deposition method [17]. By use of transient absorption spectroscopy SF was observed in perylene-3,4,:9:10-bis(dicarboximide) (PDI) covalently linked with $\beta$-apocarotene (Car) in two-step process: fast triplet formation in the PDI-Car dyad with subsequent triplet-triplet energy transfer to the Car triplet state [18].

\section{Singlet fission versus triplet fusion}

Triplet fusion or triplet-triplet annihilation is a process reversed to SF, when two neighboring molecules in their triplet states interact with formation of one singlet exciton and the other one in the ground state. Triplet fusion leads to delayed fluorescence $[19,20]$ and may lead to a delayed SF.

\section{On the mechanism of SF}

Detailed mechanism of SF is not yet resolved. Direct SF has been implicated, whereby the Coulomb potential yields a direct interaction between $S_{1}$ and correlated triplet pair TT, avoiding any intermediates $[20,21]$.

Indirect SF assumes existence of an intermediate state. This intermediate state was considered (i) to have multiexcitonic character, (ii) to be energetically accessible from the optically allowed excited state, and (iii) able to efficiently dissociate into multiple electron-hole pairs $[22,23]$.

Intermediate states such as charge-transfer state [24], "multiexciton state" [22,25], "dark state" [26], "doubly excited state" [27] or excimer state [16] have been reported.

\section{References}

1. Singh S, Jones WJ, Siebrand W, Stoicheff BP, Schneider WG (1965) J Chem Phys 42: 330 .

2. Geacintov N, Pope M, Vogel F (1969) Effect of Magnetic Field on the Fluorescence of Tetracene Crystals: Exciton Fission. Phys Rev Lett 22: 593.

3. Merrifield RE, Avakian P, Groff RP (1969) Fission of singlet excitons into pairs of triplet excitons in tetracene crystals. Chem Phys Lett 3: 386-388.

4. Smith MB, Michl J (2010) Singlet fission. Chem Rev 110: 6891-6936.

5. Smith MB, Michl J (2013) Recent advances in singlet fission. Annu Rev Phys Chem 64: 361-386.

6. Ma L, Zhang K, Kloc C, Sun H, Michel-Beyerle ME, et al. (2012) Singlet fission in rubrene single crystal: direct observation by femtosecond pumpprobe spectroscopy. Phys Chem Chem Phys 14: 8307-8312.

7. Johnson JC, Nozik AJ, Michl J (2010) High triplet yield from singlet fission in a thin film of 1,3-diphenylisobenzofuran. J Am Chem Soc 132: 16302-16303.

8. Wilson MW, Rao A, Clark J, Kumar RS, Brida D, et al. (2011) Ultrafast dynamics of exciton fission in polycrystalline pentacene. J Am Chem Soc 133: 11830-11833.

9. Ma L, Zhang K, Kloc C, Sun H, Soci C, Michel-Beyerle ME, Gurzadyan GG (2013) Fluorescence from rubrene single crystals: Interplay of singlet fission and energy trapping. Phys Rev B 87: 201-203.

10. Wilson MW, Rao A, Johnson K, Gélinas S, di Pietro R, et al. (2013) Temperature-independent singlet exciton fission in tetracene. J Am Chem Soc 135: 16680-16688.

11. Ma L, Galstyan G, Zhang K, Kloc C, Sun H, et al. (2013) Two-photoninduced singlet fission in rubrene single crystal. J Chem Phys 138: 184508.

12. Ma L, Tan KJ, Jiang H, Kloc C, Michel-Beyerle ME, et al. (2014) Excitedstate dynamics in an $\hat{\mathrm{I}} \pm$-perylene single crystal: two-photon- and consecutive two-quantum-induced singlet fission. J Phys Chem A 118: 838-843.

13. Albrecht WG, Michel-Beyerle ME, Yakhot V (1978) Exciton Fission in Excimer Forming Crystal. Dynamics of an Excimer Build-Up in a-Perylene Chem Phys 35: 193-200.

14. Bakulin AA, Morgan SE, Kehoe TB, Wilson MWB, Chin AW, et al. (2016) Real-time observation of multiexcitonic states in ultrafast singlet fission using coherent 2D electronic spectroscopy. Nature Chem 8: 16-23.

15. Wan Y, Guo Z, Zhu T, Yan S, Johnson J, et al. (2015) Cooperative singlet and triplet exciton transport in tetracene crystals visualized by ultrafast microscopy. Nat Chem 7: 785-792.

16. Walker BJ, Musser AJ, Beljonne D, Friend RH (2013) Singlet exciton fission in solution. Nat Chem 5: 1019-1024.

17. Zirzlmeier J, Lehnherr D, Coto PB, Chernick ET, Casillas R, et al. (2015) Singlet fission in pentacene dimers. Proc Natl Acad Sci U S A 112: 5325-5330.

18. Mauck CM, Brown KE, Horwitz NE, Wasielewski MR (2015) Fast Triplet Formation via Singlet Exciton Fission in a Covalent Perylenediimide- $\beta$ apocarotene Dyad Aggregate. J Phys Chem A 119: 5587-5596.

19. Ryasnyanskiy A, Biaggio I (2011) Triplet exciton dynamics in rubrene single crystals. Phys Rev B 84: 193-203.

20. Burdett JJ, Bardeen CJ (2012) Quantum beats in crystalline tetracene delayed fluorescence due to triplet pair coherences produced by direct singlet fission. J Am Chem Soc 134: 8597-8607.

21. Ma L, Kloc C, Soci C, Michel-Beyerle ME, Gurzadyan GG (2014) Singlet fission in organic crystals. In: Gurzadyan GG (ed.) "Ultrafast dynamics in molecules, nanostructures and interfaces", World Scientific ISBN-10: 9814556912. 
Citation: Gurzadyan GG (2016) Femtosecond Laser Spectroscopy and Singlet Fission. J Laser Opt Photonics 3: e109. doi:10.4172/2469-410X. $1000 \mathrm{e} 109$

Page 3 of 3

22. Zimmerman PM, Zhang Z, Musgrave CB (2010) Singlet fission in pentacene through multi-exciton quantum states. Nat Chem 2: 648-652.

23. Chan WL, Berkelbach TC, Provorse MR, Monahan NR, Tritsch JR, et al. (2013) The quantum coherent mechanism for singlet fission: experiment and theory. Acc Chem Res 46: 1321-1329.

24. Monahan N, Zhu XY (2015) Charge transfer-mediated singlet fission. Annu Rev Phys Chem 66: 601-618.

25. Chan WL, Ligges M, Zhu XY (2012) The energy barrier in singlet fission can be overcome through coherent coupling and entropic gain. Nat Chem 4: $840-845$.
26. Burdett JJ, Gosztola D, Bardeen CJ (2011) The dependence of singlet exciton relaxation on excitation density and temperature in polycrystalline tetracene thin films: kinetic evidence for a dark intermediate state and implications for singlet fission. J Chem Phys 135: 214508.

27. Teichen PE, Eaves JD (2012) A microscopic model of singlet fission. J Phys Chem B 116: 11473-11481. 